\title{
TRATAMIENTO CON FÉRULA DENTAL EN BRUXISMO INDUCIDO POR ANTICONVULSIVO: REPORTE DE CASO
}

\section{DENTAL SPLINT TREATMENT IN BRUXISM INDUCED BY ANTI-SEIZURE DRUG: CASE REPORT}

\section{Casimira Valeria Chuquimez-Ventura' * (i) valeriachuquimez@icloud.com \\ Wendoly Rosalinda Alfaro-Escalante ${ }^{1 \mathbb{D}}$ wendoly.alfaro28@gmail.com}

Artículo recibido: 01/03/2020 Arbitrado por pares Artículo aceptado: 14/04/2020

\section{* Autor corresponsal:}

Casimira Valeria

Chuquimez-Ventura

valeriachuquimez@icloud.com

Citar como: Chuquimez-Ventura CV, Alfaro-Escalante WR. Tratamiento con férula dental en bruxismo inducido por anticonvulsivo: reporte de caso. Rev Cient Odontol (Lima) 2020; 8(1): e012.

DOI: $10.21142 / 2523-2754-0801-2020-012$

\section{RESUMEN}

Los signos de desgaste y rechinamiento de los dientes están asociados con el bruxismo y se observan comúnmente en los consultorios dentales. En este reporte, las autoras describen un caso de bruxismo probablemente inducido por el medicamento anticonvulsivo Valpromed ${ }^{\circledR}$, que fue tratado con éxito mediante una férula dental. El paciente llegó a la clínica declarando que deseaba mejorar su estética. En el examen clínico, se observó un desgaste moderado de las superficies oclusales de los molares asociado con antecedentes de rechinamiento nocturno, característico del bruxismo del sueño. Hace aproximadamente 5 meses, el paciente comenzó la terapia con Valpromed ${ }^{\circledR}$ por orden del neurólogo, debido a episodios de migraña. El medicamento se prescribió para evitar el riesgo de convulsiones ya que el paciente había sufrido de neurocisticercosis durante la infancia. Según los consejos del especialista en rehabilitación oral y la literatura revisada, las autoras concluyeron que el bruxismo podía atribuirse a la terapia con el medicamento anticonvulsivo. Para el tratamiento, se escogió una técnica mínimamente invasiva. Aunque el paciente todavía está en controles, la férula dental parece ser un enfoque prometedor para el tratamiento de esta afección.

Palabras clave: rehabilitación oral, bruxismo, anticonvulsivos, férula dental

\section{ABSTRACT}

Signs of tooth wear and grinding are associated with bruxism, which is commonly observed in dental offices. Here, the authors describe a case of bruxism probably induced by the anti-seizure drug Valpromed ${ }^{\circledR}$, that was successfully treated with a dental splint. A man was attended in the dental clinic for improvement of dental aesthetics. On clinical examination moderate overwear of the occlusal surfaces of molars was observed associated with a history of nightly grinding, characteristic of sleep bruxism. Approximately 5 months previously, the patient had started therapy with Valpromed ${ }^{\circledR}$ due to episodes of migraine. The drug had been prescribed by a neurologist to prevent convulsions since the patient had had neurocysticercosis during childhood. Based on the advice of an oral rehabilitation specialist and the literature reviewed, it was concluded that the presence of bruxism in this patient may be attributed to the therapy with the anti-seizure medication. Although the patient is still under controls, the dental splint seems to be a promising approach for the treatment of this condition. The patient was treated with a minimally invasive dental splint and is monitored periodically, showing good results.

Keywords: Oral rehabilitation, bruxism, anti-siezure drug, dental splint

\footnotetext{
1 Facultad de Odontología, Universidad Nacional Federico Villarreal. Lima, Perú.
} 


\section{INTRODUCCIÓN}

Según el enfoque actual, el bruxismo es considerado una actividad parafuncional asociada con el sistema estomatognático y que incluye la masticación de labios y mejillas, la mordida de uñas y el apretamiento de los dientes. Asimismo, el bruxismo puede clasificarse en dos tipos: bruxismo de vigilia (BV) y del sueño (BS) (1); este último es el tema del presente reporte de caso. E1 bruxismo del sueño se define como una actividad muscular masticatoria - no considerado como un trastorno del sueño- que se caracteriza por ser rítmico o no rítmico ${ }^{(2)}$. Entre los efectos de esta parafunción se asocian el dolor orofacial, los desórdenes temporomandibulares, el desgaste dental, las fracturas en las restauraciones, así como los daños en otros componentes bucales, faciales y cervicales debido a los movimientos de apretar y rechinar las piezas dentarias $\left({ }^{3}\right)$.

Desde el punto de vista clínico, el análisis del apretamiento de las piezas dentarias superiores contra las inferiores es crucial para el diagnóstico de esta patología, ya que se genera desgaste y se altera la oclusión en conjunto con la función cráneo-mandibular. Por lo tanto, es de suma importancia realizar un adecuado diagnóstico clínico y tratar la parafunción antes de reconstruir la oclusión. En ese sentido, el tratamiento con férulas dentales ha demostrado éxito en frenar la parafunción y el desgaste dental, ya que su uso evita la sobrecarga de fuerzas y alivia el desbalance de las patologías cráneomandibulares que pueden haberse originado producto del bruxismo $\left({ }^{4}\right)$.

Se han llevado a cabo numerosos estudios para definir la etiología de esta parafunción, sin mayor éxito. Diversas hipótesis la relacionan con factores psicológicos $\left(^{5}\right)$, neurológicos y morfológicos (6,7). Reportes recientes consideran también que el BS es resultado de factores tanto patofisiológicos como genéticos $\left.{ }^{8}\right)$, así como de factores externos como el abuso de drogas y medicamentos psicotrópicos, entre los que podemos mencionar los antidepresivos, antipsicóticos, ansiolíticos y anticonvulsivantes $\left({ }^{9}\right)$. El Valpromed ${ }^{\circledR}$ o ácido valproico es un anticonvulsivante que actúa aumentando los ni-veles cerebrales de GABA, disminuye los de amino-ácidos excitadores y modifica la conductancia del potasio. Está indicado para epilepsias generalizadas, parciales y complejas; además, en el tratamiento de episodios maníacos asociados con el trastorno bipolar $\left({ }^{10}\right)$.

Investigaciones previas mencionan que el bruxismo puede ser considerado común durante la terapia con medicamentos psicotrópicos, incluyendo antipsicóticos y antidepresivos $\left({ }^{11}\right)$. Sin embargo, no hay reportes de casos de bruxismo del sueño asociados con la ingesta de Valpromed $^{\circledR}$ en bases de datos de revistas indexadas. El objetivo de este artículo es describir el manejo clínico de un caso de bruxismo asociado con la ingesta de anticonvulsivantes a través de un tratamiento mínimamente invasivo como la férula dental.

\section{REPORTE DE CASO}

\section{ETAPA CLÍNICA DIAGNÓSTICA}

El paciente de 50 años, de género masculino, buscó atención odontológica por inconformidad estética y masticatoria. Durante la anamnesis, refirió que hace aproximadamente 5 meses inició el consumo de Valpromed $^{\circledR}$ de $500 \mathrm{mg}$, una vez por día, por indicación del neurólogo. También manifestó que su esposa le mencionó que rechinaba los dientes mientras dormía.

Durante el examen clínico extraoral, manifestó músculo masetero hipertónico y doloroso a la palpación, y chasquido del ATM derecho al cierre sin dolor a la palpación. En el examen clínico intraoral, se observó que presentaba desgaste dental localizado en zona anteroinferior, sin pérdida de la dimensión vertical oclusal, mordida invertida de las hemiarcadas derechas, recesión gingival en piezas 1.8, 2.3, 3.4,3.5,3.6, 4.7, 4.4, y trauma oclusal primario (figs. 1 y 2 ).

En el examen radiográfico, se observó asimetría en ambos lados de la articulación temporomandibular; el cóndilo derecho es más pequeño y se sitúa más alejado de la cavidad glenoidea en comparación con el cóndilo izquierdo (fig. 3). 


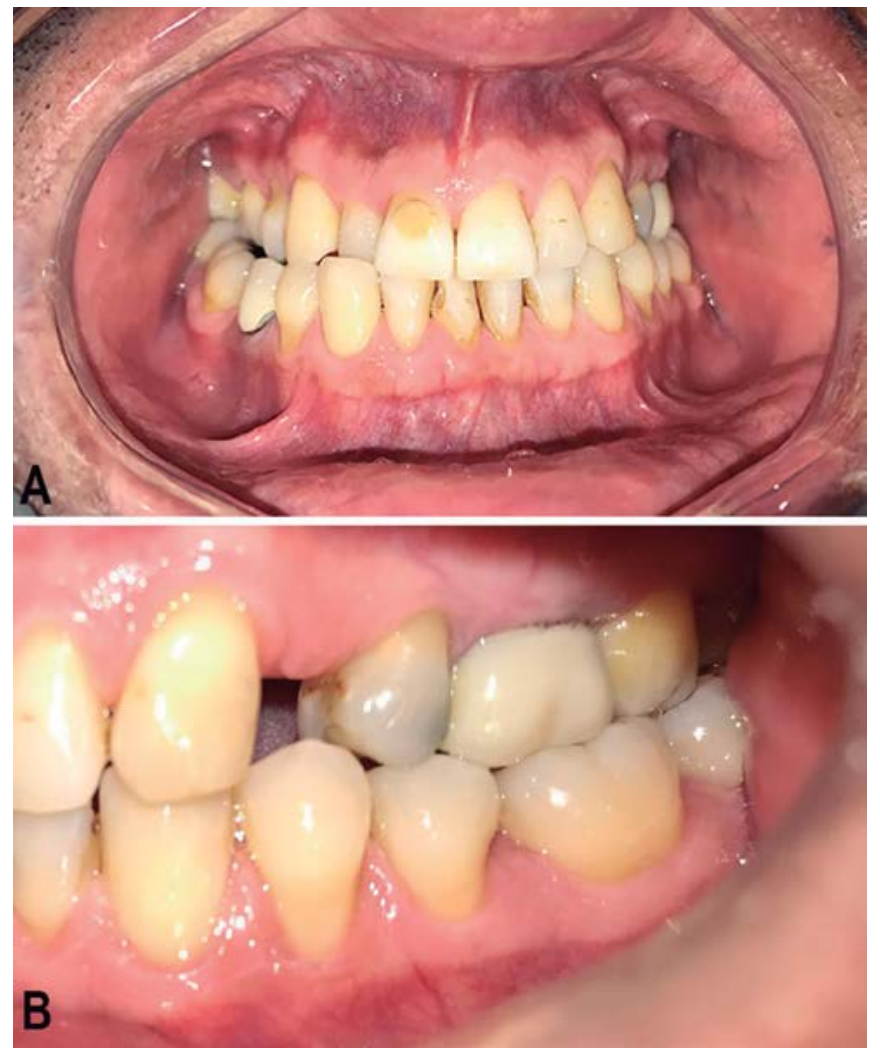

Figura I. A) Fotografía intraoral inicial que muestra mordida invertida de las hemiarcadas derecha. B) Recesión gingival en las piezas 3.4 y 3.5 .
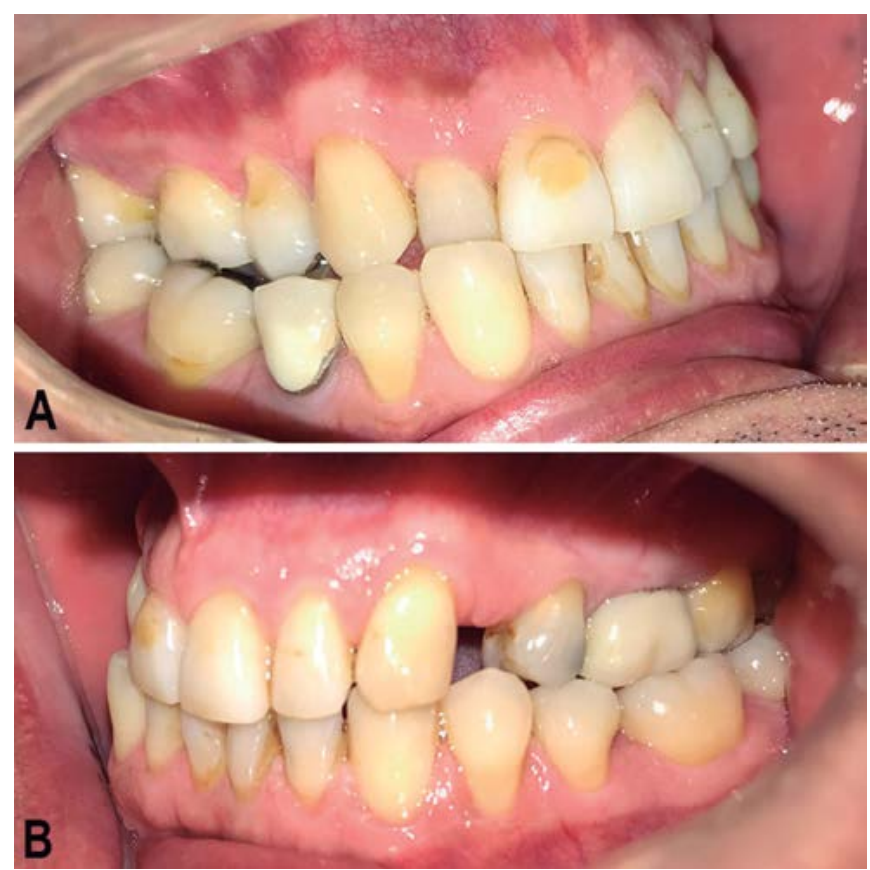

Figura 2. Fotografías intraorales. A) Vista lateral derecha. B) Vista lateral izquierda.

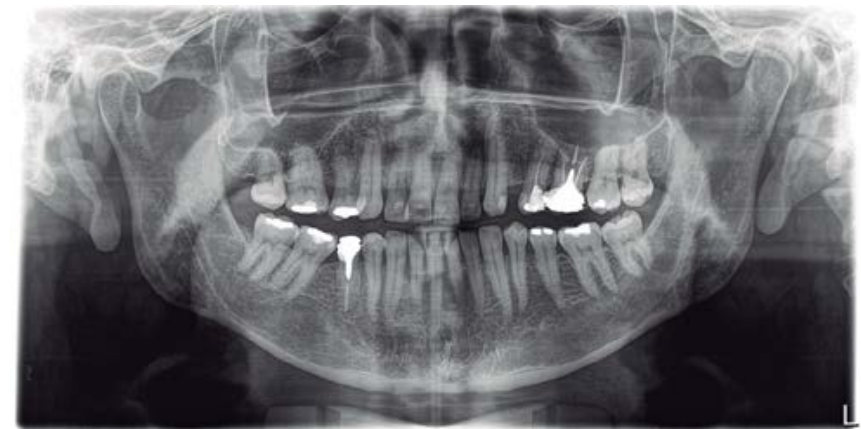

Figura 3. Radiografía panorámica.

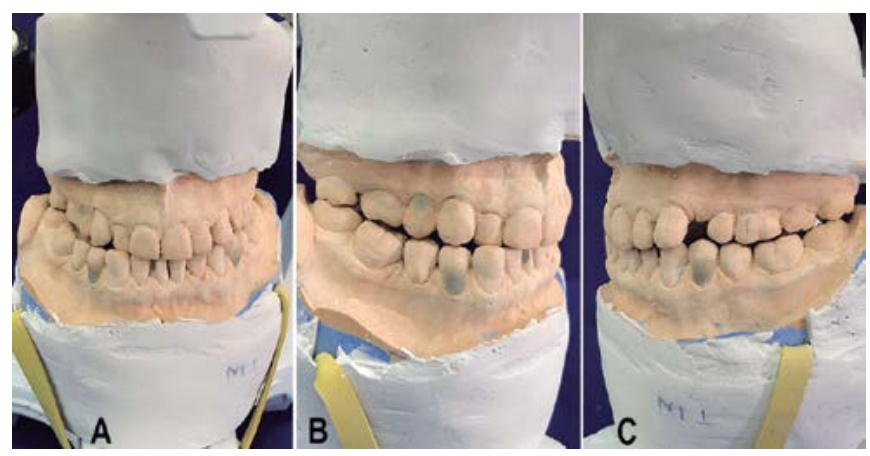

Figura 4. A) Montaje en máxima intercuspidación en articulador Bio-Art A7 Plus. B) Fotografía lateral derecha de montaje de modelos en máxima intercuspidación. Curva de Speed: asimétrica por piezas extruidas, se aprecia extrusión de pieza 4.3. Curva de Wilson: asimétrica en el lado derecho por la intrusión de pieza 4.7. C) Fotografía lateral izquierda de montaje de modelos en máxima intercuspidación. Curva de Speed: asimétrica por piezas extruidas, se aprecia extrusión de pieza 3.4 y 3.5 .

\section{ETAPA CLÍNICA}

Inicialmente, se elaboraron 3 modelos: 2 de estudio y 1 de trabajo. A continuación, los modelos de estudio fueron montados en articuladores Bio-Art A7 Plus (Bio-Art, São Paulo, Brasil) y se proyectó un registro en relación céntrica usando la técnica chin point o guía del mentón $\left({ }^{12}\right)$, y otro en máxima intercuspidación (fig. 4).

Con la ayuda de ambos modelos de estudio, se planificaron las alturas de los dientes anteriores con desgaste y la reorganización de la oclusión. En el modelo de trabajo, se realizó un encerado para la confección de la férula dental de resina acrílica, puesto que en estos casos se considera como primer tratamiento antes de la rehabilitación definitiva.

La férula dental, también llamada férula oclusal superior permisiva, fue confeccionada e instalada con los patrones 


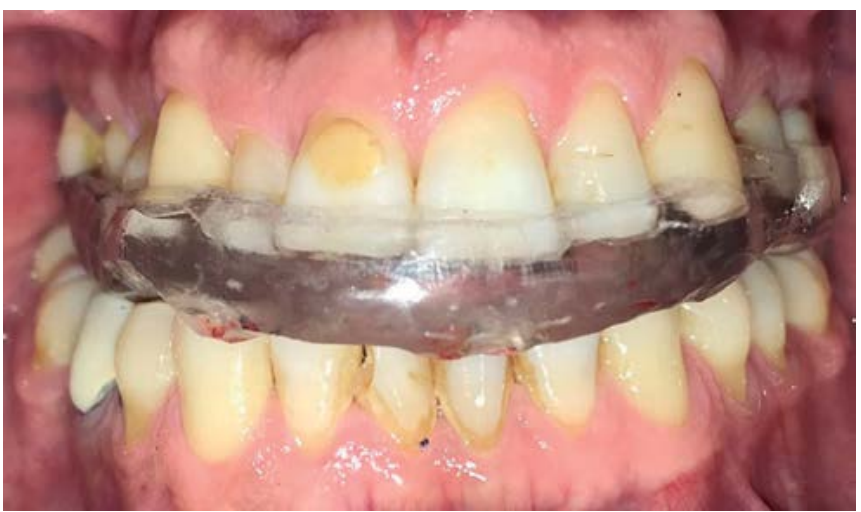

Figura 5. Férula dental instalada.

de oclusión ideales, mediante el uso de papel articular. Al paciente se le indicó el uso nocturno (fig. 5).

La férula dental debe ser usada hasta que se haya logrado la remisión de los síntomas dolorosos, la verificación de la relación céntrica y la estabilidad de la mordida con la férula en el curso del algunos días. El promedio del tratamiento con férula para estos tipos de trastornos es de 2 a 4 semanas para alcanzar una oclusión estable $\left({ }^{13}\right)$.

Con relación al seguimiento, el primer control del uso de la férula dental se realizó a los 7 días de la instalación. El paciente refirió disminución del dolor muscular y leve incomodidad al dormir. Dos semanas después de la instalación, el paciente refirió no tener dolor muscular, pero sí leve incomodidad por el uso del aparato. Es por ello que, al término de la evaluación, el paciente se consideró asintomático y listo para la rehabilitación definitiva.

\section{DISCUSIÓN}

El tratamiento para el desgaste dental como signo del bruxismo del sueño es fundamental para evitar daños más severos en el sistema estomatognático. En el presente caso clínico, se consideró el tratamiento del rechinamiento nocturno con una férula dental como el paso previo al inicio de las restauraciones, ya que el pronóstico podría verse afectado de no tratarse primero la causa que origina el desgaste $\left({ }^{14}\right)$.
La etiología del bruxismo del sueño es multifactorial. Los antecedentes sugieren que niveles altos de ansiedad podrían ser las causas principales por el estímulo que se produce en el sistema nervioso central, lo que altera la producción de dopamina y causa la atrición y rechinamiento de las piezas dentarias $(6,14)$. Sin embargo, existen factores sistémicos o externos también asociados al desencadenamiento de esta patología, como los medicamentos psicotrópicos. Se ha identificado que la dopamina y los fármacos antidepresivos que actúan sobre la neurotransmisión de serotonina influyen críticamente en la patogénesis del bruxismo inducido farmacológicamente, así como aquellos fármacos antihistaminérgicos, que resultan inductores del bruxismo por su efecto desinhibidor del sistema serotoninérgico $\left.{ }^{(15}\right)$.

Entre los pocos casos en los que se reporta el rechinamiento dental nocturno en pacientes con epilepsia, esta se produce por compromiso del lóbulo temporal $(7,8)$. El paciente del caso descrito presenta antecedentes de microconvulsiones durante su infancia, debido a neurocisticercosis, las cuales remitieron después de completar el tratamiento para esa enfermedad. Estas microconvulsiones representaban epilepsia relacionada con el automatismo oromandibular, ya que los episodios incluían temblores de la mandíbula. Recientemente, el paciente acudió al servicio de neurología por presentar episodios recurrentes de migraña como secuela del daño neuronal, motivo por el cual se le prescribió Valpromed ${ }^{\circledR}$ (ácido valproico) para evitar posibles episodios de epilepsia. Por lo tanto, en este caso, la causa de las convulsiones podría ser diferente de la de los antecedentes, ya que no se trata de alguna alteración funcional del lóbulo temporal, como comúnmente ocurre, sino por las secuelas neurológicas de la cisticerosis $\left.{ }^{7}\right)$. Sin embargo, el paciente refiere que, a raíz de la prescripción, experimenta rechinamiento nocturno y dolor muscular orofacial, con lo cual fue diagnosticado como bruxista del sueño. El bruxismo aparentemente inducido por la toma de Valpromed ${ }^{\circledR}$ resulta particularmente interesante, ya que se contrapone a lo reportado por Lin y Tang $\left({ }^{16}\right)$, quienes de forma accidental encontraron que el valproato de sodio, una sal 
sódica del ácido valproico prescrita para el tratamiento de un paciente con trastorno bipolar, pudo tratar efectivamente el bruxismo del sueño. En nuestro reporte, por el contrario, la toma de ácido valproico se relaciona con el desencadenamiento del bruxismo nocturno. Esto podría explicarse porque ambos fármacos no tienen la misma composición química y por el hecho de presentar antecedentes de cisticercosis, que podrían haber influido en la respuesta del paciente a compuestos similares $\left({ }^{16}\right)$.

Varios autores afirman que el bruxismo del sueño, antes de ser considerado una condición patológica, debe aceptarse como una condición controlada siempre que se tome en cuenta la influencia de factores de riesgo sistémicos que pueda presentar el paciente. Se afirma incluso que el bruxismo por sí mismo no requiere tratamiento y que este debería indicarse solo cuando se presenten problemas asociados $\left({ }^{17}\right)$. En el presente caso, el paciente ya presentaba dolor a causa de la hipertonicidad del músculo masetero, chasquido en la articulación temporomandibular y disconformidad estética, lo cual fue suficiente para intervenir el cuadro de bruxismo del sueño antes de la rehabilitación estética y funcional del paciente. El uso de una férula dental es el tratamiento elegido por los especialistas y odontólogos generales ya que es mínimamente invasivo en comparación con un proceso de ajuste oclusal. Sin embargo, debe considerarse que su indicación no puede ser generalizada para todos los casos. Al respecto, Candirli et al. $\left({ }^{18}\right)$ descubrieron que el conocimiento de los dentistas sobre el tratamiento del bruxismo y los desórdenes temporomandibulares con férulas oclusales es insuficiente. Esto es importante ya que se recomienda que los pacientes con patologías de la oclusión sean derivados a un especialista en rehabilitación oral y que estos casos sean estudiados con las herramientas adecuadas, con decisiones basadas en el análisis de la oclusión mediante los modelos de estudio en articuladores semiajustables. A pesar de las múltiples estrategias existentes para el manejo del paciente bruxista del sueño, no se ha llegado a un consenso para determinar cuál es el tratamiento definitivo $\left({ }^{19}\right)$. Por lo tanto, es importante considerar la historia clínica y los factores externos que pueden desencadenar el cuadro doloroso asociado y aplicar estrategias mínimamente invasivas, como las férulas dentales, previamente a todo tratamiento restaurador, a fin de garantizar el éxito del tratamiento y el bienestar del paciente.

\section{CONCLUSIÓN}

Si bien existen evidencias acerca de la influencia de los medicamentos psicotrópicos en el bruxismo del sueño, no se han encontrado antecedentes específicos para el Valpromed ${ }^{\circledR}$. En el presente caso, al no conocer la etiología del bruxismo del sueño y no tener un tratamiento establecido que garantice el 100\% de efectividad; se recomendó controlar la parafunción mediante una férula dental antes de la rehabilitación. Por lo tanto, el tratamiento fue mínimamente invasivo y orientado a controlar la parafunción y reorganizar la oclusión. Una vez estabilizado el rechinamiento nocturno, se realizará el tratamiento rehabilitador integral, funcional y estético.

\section{AGRADECIMIENTOS}

Las autoras agradecen al Dr. Hugo Pérez por las enseñanzas, asesoramiento y supervisión durante el tratamiento clínico, y al paciente $\mathrm{M}$. $\mathrm{CH}$. B por la confianza y su responsabilidad al acudir siempre a consulta y seguir las indicaciones.

Contribución de autoría: Casimira Valeria ChuquimezVentura y Wendoly Alfaro-Escalante realizaron ambas los procedimientos clínicos y redactaron el manuscrito.

Fuentes de financiamiento: Autofinanciado.

Potenciales conflictos de interés: Las autoras declaran que no existe conflicto de interés. 


\section{REFERENCIAS BIBLIOGRÁFICAS}

1. Reddy SV, Kumar MP, Sravanthi D, Mohsin AH, Anuhya V. Bruxism: a literature review.J Int Oral Health. 2014; 6 (6): 105-9.

2. Lobbezoo F, Ahlberg J, Raphael KG, Wetselaar P, Glaros AG, Kato $\mathrm{T}$, et al. International consensus on the assessment of bruxism: Report of a work in progress. J Oral Rehabil. 2018; 45: 837-44. doi: 10.1111/joor.12663

3. Morales SY,Neri ZF, Castellanos JL. Fisiopatología del bruxismo nocturno. Factores endógenos y exógenos. Rev ADM. 2015; 72 (2): 78-84.

4. Hirai K, Ikawa T, Shigeta Y, Shigemoto S, Ogawa T. Evaluation of sleep bruxism with a novel designed occlusal splint. J Prosthodont Res. 2017; 61 (3): 333-43. doi: 10.1016/j.jpor.2016.12.007

5. Montero J, Gómez-Polo C. Personality traits and dental anxiety in self-reported bruxism. A cross-sectional study. J Dent. 2017; 65: 45-50. doi: 10.1016/j.jdent.2017.07.002

6. Shen Z, Zhou N, Wu L. A preliminary study on potential association between psychopathological status and rhythmic masticatory muscle activity of young patients with sleep bruxism in Tianjin, China. Sleep Med. 2018; 51: 99-104.

7. Bušková J, Koštálová J, Miletínová E, Bušek P. Comorbid episodes of primary bruxism and bruxism as an epileptic activityrelated motor event. Sleep Med. 2019; 64: 1-2.

8. De Castilho LS, Abreu MH, De Oliveira RB, Souza E, Silva $\mathrm{ME}$, Resende VL. Factors associated with mouth breathing in children with -developmental -disabilities. Spec Care Dentist. 2016; 36 (2): 75-9. doi: 10.1111/scd.12157

9. Melo G, Dutra KL, Rodrigues Filho R, et al. Association between psychotropic medications and presence of sleep bruxism: A systematic review. J Oral Rehabil. 2018; 45 (7): 545-54.
10. Vidal Vademecum Consult [Internet]. Madrid: Vidal Group; 2018. Disponible en https://www.vademecum.es/productosvademecum-vidal+vademecum+consult-44

11. Uca AU, Uğuz F, Kozak HH, et al. Antidepressant-induced sleep bruxism: prevalence, incidence, and related factors. Clin Neuropharmacol. 2015; 38 (6): 227-30.

12. Caravadossi A, Guadalupe M, Odizzio S, Rué G, Vidal A, Villarnobo F, et al. Métodos de registro de la relación céntrica. ¿Son una necesidad en el diagnóstico y tratamiento de ortodoncia? Actas Odontol. 2012; 9 (11): 59-64.

13. Dawson PE. Oclusión funcional: diseño de la sonrisa a partir de la ATM. Caracas: Amolca; 2009.

14. Wetselaar P, Vermaire J, Visscher C, Lobbezoo F. The prevalence of tooth wear in the dutch adult population. Caries Res. 2016; 50 : 543-50. doi: 10.5177/ntvt.2018.04.17201

15. Falisi G, Rastelli C, Panti F, Maglione H, Quezada Arcega R. Psychotropic drugs and bruxism. Expert Opin Drug Saf. 2014; 13 (10): 1319-26.

16. Lin XL, Tang SY. Sodium valproate may be a treatment for sleep bruxism. J Child Adolesc Psychopharmacol. 2013; 23 (9): 636-7.

17. Beddis H, Pemberton M, Davies S. Sleep bruxism: an overview for clinicians. Br Dent J. 2018; 225 (6): 497-501.

18. Candirli C, Korkmaz YT, Celikoglu M, Altintas SH, Coskun U, Memis S. Dentists' knowledge of occlusal splint therapy for bruxism and temporomandibular joint disorders. Niger J Clin Pract. 2016; 19 (4): 496-501.

19. Yap AU, Chua AP. Sleep bruxism: Current knowledge and contemporary management. J Conserv Dent. 2016; 19 (5): 3839. doi: 10.4103/0972-0707.190007 